Bundesgesundheitsbl 2016 $59: 514-522$ DOI 10.1007/s00103-016-2323-x

Online publiziert: 15. März 2016

(c) The Author(s) 2016 . This article is available at SpringerLink with Open Access

CrossMark

\author{
Paulus Kirchhof ${ }^{1,2,3,7}$. Andreas Goette ${ }^{3,4}$ - Michael Näbauer ${ }^{3,5}$ - Ulrich Schotten ${ }^{3,6}$ \\ 'Institute of Cardiovascular Sciences, University of Birmingham and SWBH and UHB NHS Trusts, \\ Birmingham, UK \\ ${ }^{2}$ Department für Kardiologie und Angiologie, Universitätsklinikum Münster, Münster, Deutschland \\ ${ }^{3}$ AFNET e. V., Münster, Deutschland \\ ${ }^{4}$ Vincenz-Krankenhaus, Paderborn, Deutschland \\ ${ }^{5}$ Klinikum der Ludwig Maximilian Universität, München, Deutschland \\ ${ }^{6}$ Department of Physiology, Maastricht University, Maastricht, Niederlande \\ ${ }^{7}$ Kompetenznetz Vorhofflimmern, Münster, Deutschland
}

\title{
12 Jahre AFNET. Vom Forschungsnetzwerk zur Academic Research Organisation
}

Herzrhythmuserkrankung substanziell zu verbessern.

Vorhofflimmern ist die häufigste anhaltende Herzrhythmusstörung. Bei Vorhofflimmern liegt eine chaotische Aktivierung der Vorhöfe vor, die zu einem Verlust der Kontraktion der Vorhöfe und zu einer unregelmäßigen Aktivierung der Herzkammern („absolute Arrhythmie“) führt. Vorhofflimmern kann schwere Folgen haben: etwa Schlaganfälle, Herzinsuffizienz, oder kardiale Todesfälle. In Deutschland leben rund 2 Mio. Patienten mit Vorhofflimmern. Betroffen sind v. a. ältere Menschen. Aufgrund des demografischen Wandels wird sich die Zahl der Vorhofflimmerpatienten in den nächsten 40 Jahren mehr als verdoppeln. Trotz beachtlicher Fortschritte bei der Forschung und Behandlung von Vorhofflimmern sind weiterhin viele Fragen bei der Therapie von Vorhofflimmern offen. So wird z. B. die Übersterblichkeit, die mit Vorhofflimmern assoziiert ist, auch bei bestem evidenzbasiertem Management nur z. T. reduziert. Seit seiner Gründung im Jahr 2003 ist es das Ziel des Kompetenznetzes Vorhofflimmern (Atrial Fibrillation Network [AFNET]), die Forschung zu Vorhofflimmern in Deutschland zu vernetzen und zu stärken und durch die Translation von neuen Erkenntnissen in neue Diagnostik- und Therapieverfahren, die Behandlung von Patienten mit dieser

\section{„Das Ganze ist mehr als die Summe seiner Teile" (Aristoteles)}

Das Kompetenznetz Vorhofflimmern wurde als eines von 3 kardiovaskulären Kompetenznetzen in der Medizin im Jahr 2002 in einem 2-stufigen Antragsverfahren zur Förderung ausgewählt. Die Kompetenznetze in der Medizin waren ein Förderinstrument des BMBF, das spezifisch zur Stützung von translationaler, patientenorientierter Forschung in Deutschland eingerichtet wurde. Insgesamt wurden 17 Kompetenznetze gefördert. Der Netzwerkcharakter des AFNET spiegelte sich in der initialen Organisationsform wider: Das Netz bestand aus 3 Arbeitsfeldern, nämlich Beobachtungsstudien, kontrollierten klinischen Studien und mechanistischer Forschung (siehe - Abb. 1). Neben dieser „horizontalen Vernetzung", die Wissenschaftlern, Ärzten und Experten ${ }^{1}$ mit unterschiedlichen Kenntnissen und Blickwinkeln zusammenbrachte, um Vorhofflimmern

\footnotetext{
1 Im vorliegenden Beitrag wird aus Gründen der sprachlichen Vereinfachung nur die männliche Form verwendet. Es sind jedoch stets Personen männlichen und weiblichen Geschlechts gleichermaßen gemeint.
}

aufgrund eines besseren Verständnisses besser $\mathrm{zu}$ erkennen und $\mathrm{zu}$ behandeln, ist das AFNET vom ersten Tag an „vertikal vernetzt“ angelegt und bringt universitäre Forscher und Ärzte, weitere Krankenhäuser und Arztpraxen als gleichberechtigte Zentren im Netz zusammen. Während der BMBF-Förderung wurde das AFNET regelmäßig von einem wissenschaftlichen Beirat begutachtet.

\section{Erfolgreich vernetzt: Ergebnisse der Arbeit des AFNET}

In den ersten Jahren war es eine der Hauptaufgaben des AFNET, ein Netzwerk von Studienzentren und interessierten Partnern aufzubauen und $\mathrm{zu}$ unterhalten. Dies wurde durch die gute Zusammenarbeit der Gremien und Mitglieder im Netz sowie durch die vertikale Vernetzung der AFNET-Studienzentren möglich (siehe Abb. 1). Aufgrund dieser national und international vernetzten Struktur ist es dem AFNET im Rahmen der Förderung durch das BMBF gelungen, Forschungsergebnisse aus der Grundlagenforschung in den medizinischen Versorgungsalltag $\mathrm{zu}$ transferieren. Parallel wurden führende deutsche Forschergruppen im „Bereich C“ (• Abb. 1) zusammengebracht, die neue Erkenntnisse mit einer Bedeutung für die Erkennung, Behandlung 


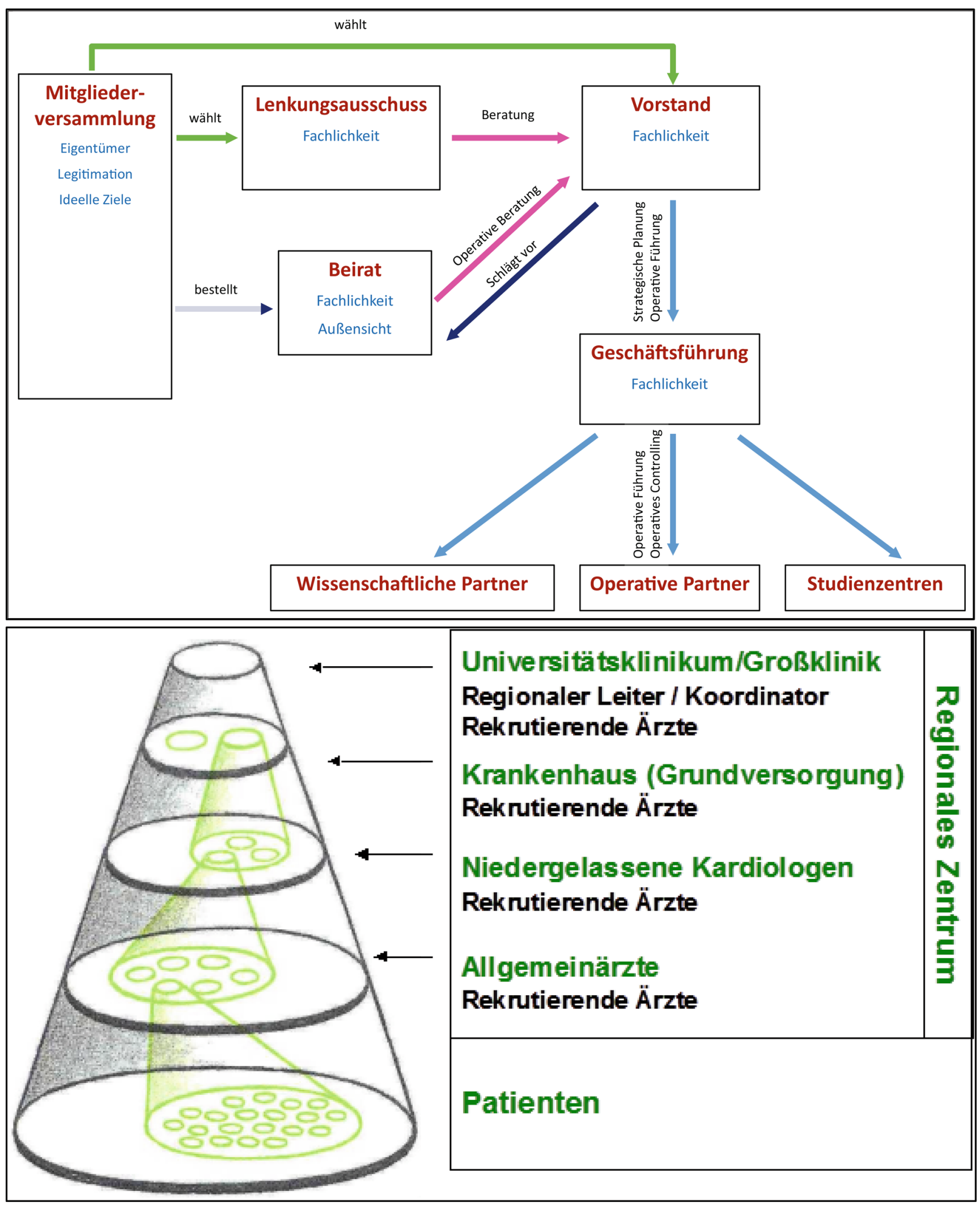

Abb. $1 \Delta$ Organigramm (oberer Teil) des AFNET und Netzwerkstruktur der AFNET-Zentren bzw. Zentrumscluster 
Bundesgesundheitsbl 2016-59:514-522 DOI 10.1007/s00103-016-2323-x

(c) The Author(s) 2016

P. Kirchhof · A. Goette · M. Näbauer · U. Schotten

\section{Jahre AFNET. Vom Forschungsnetzwerk zur Academic Research Organisation}

\section{Zusammenfassung}

„Das Ganze ist mehr als die Summe seiner Teile" (Aristoteles).

Vorhofflimmern ist die häufigste anhaltende Herzrhythmusstörung. Allein in Deutschland leben rund 2 Mio. Patienten mit Vorhofflimmern. Betroffen sind v. a. ältere Menschen. Aufgrund des demografischen Wandels wird sich die Zahl der Vorhofflimmerpatienten in den nächsten 40 Jahren mehr als verdoppeln. Die Prävalenz von Vorhofflimmern ist altersabhängig: sie beträgt in der Gesamtbevölkerung ca. $1 \%$, in der älteren Population bis zu $20 \%$. Trotz beachtlicher Fortschritte bei der Forschung und Behandlung von Vorhofflimmern sind weiterhin viele Fragen bei der Therapie von Vorhofflimmern offen. Das Ziel des Kompetenznetzes Vorhofflimmern (Atrial Fibrillation NETwork, AFNET) ist es, die
Forschung zu Vorhofflimmern auszubauen und so die Behandlung von Patienten mit dieser Herzrhythmuserkrankung substanziell zu verbessern. Hierzu haben die klinischen und experimentellen Partner ihre Anstrengungen im Kompetenznetz Vorhofflimmern gebündelt. Die enge Zusammenarbeit und die Förderung durch das Bundesministerium für Bildung und Forschung (BMBF) im Rahmen des Förderinstruments "Kompetenznetze in der Medizin“ ermöglichte so entscheidende Fortschritte in der Erkennung und Behandlung von Vorhofflimmern sowie eine deutlich verbesserte öffentliche Wahrnehmung in Deutschland und in Europa. Insbesondere hat das AFNET durch ein großes Register, multizentrische klinische kardiovaskuläre Studien und die Koordination von transla- tionaler Grundlagenforschung wesentliche Beiträge zu einem verbesserten Verständnis, früher Erkennung und besserer Therapie von Vorhofflimmern in Deutschland und Europa geleistet. Zur Sicherung dieser Erfolge hat das Kompetenznetz Vorhofflimmern bereits im Jahr 2010 den Verein Kompetenznetz Vorhofflimmern e. V. gegründet. Das AFNET hat sich zu einer "academic research organisation“ entwickelt, die internationale klinische Studien durchführt und mit dem DZHK zusammenarbeitet.

Schlüsselwörter

Vorhofflimmern · Forschungsnetzwerk . Register · Klinische Studien · Genomik

\section{AFNET. A translational research network develops into an academic research organization}

\section{Abstract}

"The whole is greater than the sum of its parts" (Aristotle).

Atrial fibrillation (AF) is the most common sustained arrhythmia and affects $1-2 \%$ of the population in developed countries, especially the elderly. We expect that the prevalence of AF will double in the next few decades. The last decades have seen important improvements in the management of atrial fibrillation, but many questions remain regarding the optimal diagnosis and management of the condition. The German Atrial Fibrillation NETwork (AFNET) was one of three cardiovascular competence networks in medicine funded

\begin{abstract}
by the German Ministry of Education and Research between 2003-2014. AFNET has contributed to the understanding of atrial fibrillation, and AFNET-led studies have led to improved clinical practices and practice guidelines in Germany and in Europe. This work has been expanded and is continuing in the AFNET association (AFNET e. V.). The AFNET association, founded in 2010 and continuing to this day, has developed into a small but fully formed academic research organisation that conducts investigatorinitiated clinical trials as the responsible sponsor in Germany, Europe, and beyond.
\end{abstract}

The AFNET association currently cooperates with EHRA (The European Heart Rhythm Association), ESC (The European Society of Cardiology) and DZHK (The German Centre for Cardiovascular Research) and receives funding from the European Union to generate evidence that can in the future lead to better prevention and management of $\mathrm{AF}$.

\section{Keywords}

Atrial fibrillation - Research network Registries · Clinical trials · Genomic research und Verhinderung von Vorhofflimmern erarbeiteten.

\section{Aufbau europäischer und internationaler Forschungs- kooperationen}

Die gemeinsame Arbeit der grundlagenorientierten Partner im AFNET hat zu vielen gemeinsamen Publikationen geführt [1-14]. Schon nach wenigen Jahren gelang es zudem AFNET-Projektleitern, internationale Forschungskonsortien aufzubauen, die u.a. von der Leducq Fondation („European/North-Ameri- can Atrial Fibrillation Research Alliance“ ENAFRA; http://pc33.uniklinikumdresden.de:8080/enafra/portal) oder der Europäischen Union („The European Network For Translational Research in Atrial Fibrillation", EUTRAF, www. eutraf.eu) gefördert wurden. Diese Forschungskonsortien profitierten sehr von der guten Zusammenarbeit der verschiedenen Gruppen im AFNET. Seit Mai 2015 ist AFNET e. V. geförderter Partner des von der Europäischen Kommission geförderten Projektes „Characterizing Atrial fibrillation by Translating its Causes into Health Modifiers in the
Elderly“ (CATCH ME, www.catch-me. info), das eine neue klinische Klassifikation von verschiedenen Formen von Vorhofflimmern entwickeln wird. Dies ist im Netz (u. a. in den AFNET/EHRAKonsensuskonferenzen) als eine wesentliche Grundlage für die Entwicklung einer personalisierten Behandlung von Vorhofflimmern identifiziert worden. Die Zusammenarbeit von Naturwissenschaftlern und klinischen Forschern im AFNET hat zudem zu neuen Therapieansätzen geführt, etwa der frühen rhythmuserhaltenden Therapie, die in der EAST - AFNET 4 Studie geprüft 


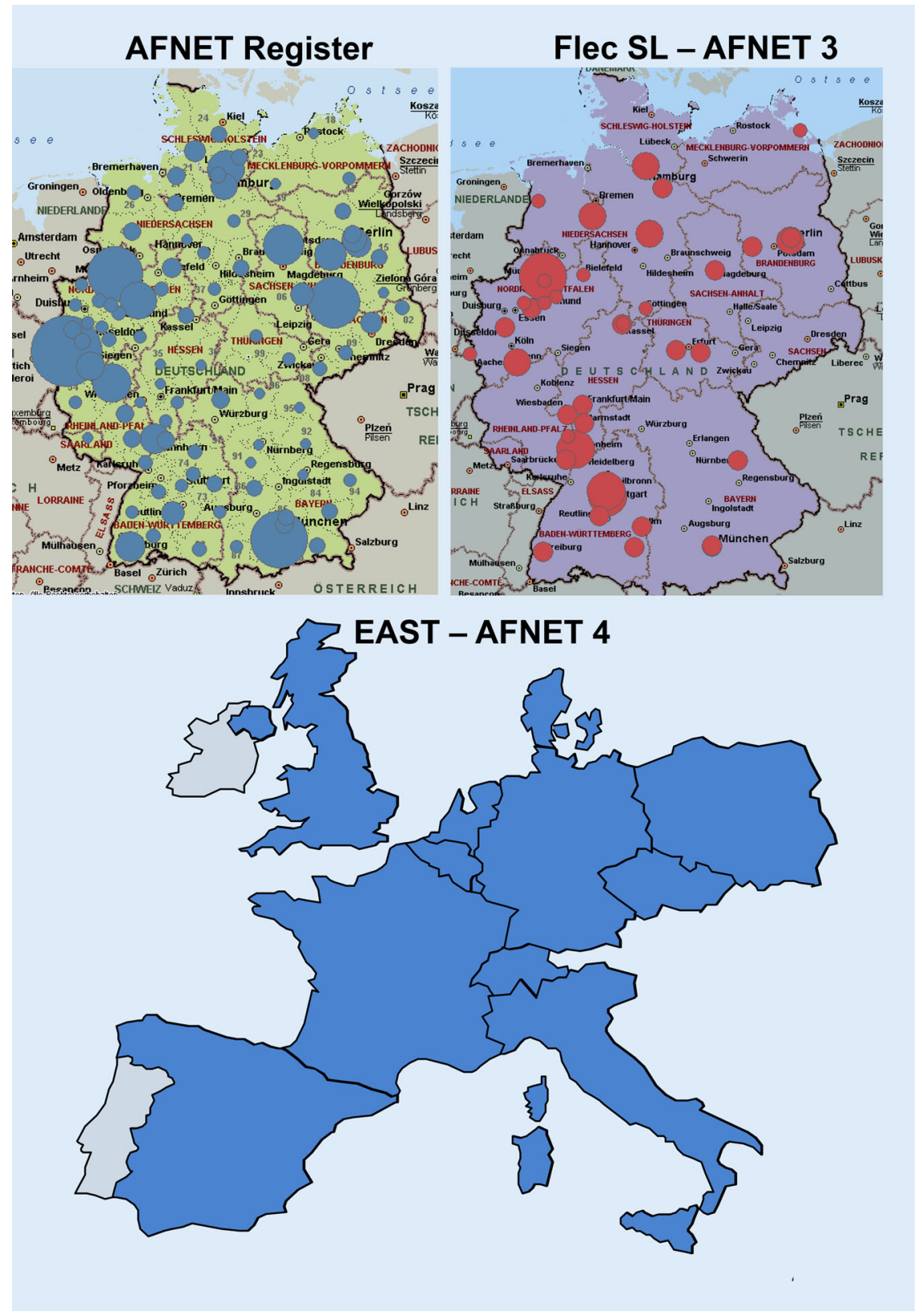

Abb. 2 \ Patienteneinschluss in ausgewählte AFNET-Studien. Oben rechts: Einschluss von Patienten in das AB1-Register. Oben links: Einschluss von Patienten in die Flec SL - AFNET 3 Studie in 44 deutschen Zentren. Unten: Einschluss von Patienten in die EAST - AFNET 4 Studie in >100 Zentren in 11 europäischen Ländern. Die Größe der Kreise ist proportional zur Patientenzahl.

wird (www.easttrial.org). Gleichzeitig wird etwa durch Zugang zu Bioproben, die in den klinischen Projekten im Netzwerk gewonnen wurden, eine relevante Grundlagenforschung ermöglicht. So haben AFNET-Forscher Bioproben seit der Gründung des AFNET zum „Cohorts for Heart and Aging Research in Genomic Epidemiology“(CHARGE AF)Konsortium beigetragen, das wesentliche Erkenntnisse über die genetischen
- AFNET 2 und Flec SL - AFNET 3 rekrutierten und die eine wichtige Basis für die deutschen Studienzentren der laufenden AFNET-Studien bilden (siehe Abb. 2), spiegeln die horizontale und vertikale Vernetzung im AFNET wider: Regionale Cluster von Rekrutierungszentren wurden für das Register von 13 regionalen Zentren gebildet. Die Aktivität dieser vertikal und horizontal vernetzten Cluster illustriert eindrücklich die Tiefe und Breite der Netzwerkaktivitäten (siehe - Tab. 1).

Die Basisdaten des Patientenkollektivs sind wissenschaftlich weitgehend ausgewertet [23-26]. Die Projektleiter arbeiten mit Hochdruck an der Auswertung der Nachverfolgungsdaten, die Informationen über Patienten mit Vorhofflimmern über viele Jahre (mittlere Nachbeobachtungsdauer $>5$ Jahre) bieten. Ein Teil der jüngeren (< 65 Jahre) Patienten, die am Register teilnehmen, gaben zusätzlich ihr Einverständnis zu einer genomischen Analyse. Diese Kohorte hat als Teil des CHARGE-AF-Konsortiums wesentlich zum Verständnis der genetischen Prädisposition zu Vorhofflimmern beigetragen.

\section{GAP AF - AFNET 1}

GAP AF - AFNET 1 ist eine prospektive, multizentrische, randomisierte klinische Studie zur Bedeutung der vollständigen Pulmonalvenenisolation bei der Katheterablation von Vorhofflimmern. Obwohl die Isolation der Pulmonalvenen seit vielen Jahren der am besten validierte Teil des Vorgehens bei Katheterablation von Vorhofflimmern ist [27], ist bis heute unklar, ob eine vollständige elektrische Isolierung der Pulmonalvenen für die klinische Effektivität erforderlich ist. Die Studie wurde von AFNET-Zentren durchgeführt. Die Ergebnisse werden in Kürze publiziert werden.

hat [15-22].

\section{AFNET-Register (AB1)}

In den Jahren 2004-2006 wurden knapp 10.000 Patienten mit Vorhofflimmern konsekutivim „AB1-Register“ des AFNET erfasst. Die Zentren im Netzwerk, die gleichzeitig auch in die kontrollierten Studien GAP-AF - AFNET 1, ANTIPAF

\section{ANTIPAF - AFNET 2}

Es ist gut belegt, dass strukturelle Veränderungen in den Vorhöfen u. a. eine Fibrose der Vorhöfe und Vorhofflimmern begünstigen. Gleichzeitig ist bekannt, dass eine Inhibition des Renin-Angiotensin-Systems mit ACE-Hemmern oder 
Tab. 1 Übersicht der Rekrutierungsleistung der regionalen Cluster im Register des AFNET (Projekt $A B 1$ )

\begin{tabular}{|llllllll}
\hline \multicolumn{2}{l}{ Patienten } & & & \multicolumn{3}{c}{ Zentren } \\
\hline Zentrum & Gesamt & $\begin{array}{l}\text { Zentrum } \\
\text { direkt }\end{array}$ & $\begin{array}{l}\text { Regionale } \\
\text { Kliniken }\end{array}$ & $\begin{array}{l}\text { Kardiolog. } \\
\text { Praxen }\end{array}$ & $\begin{array}{l}\text { Andere } \\
\text { Praxen }\end{array}$ & Gesamt Aktiv \\
\hline Aachen & 972 & 401 & 233 & 207 & 131 & 35 & 18 \\
\hline Berlin & 535 & 372 & 122 & 39 & 2 & 21 & 12 \\
\hline Bonn & 913 & 183 & 83 & 606 & 41 & 32 & 17 \\
\hline Brandenburg & 391 & 262 & 82 & 13 & 34 & 22 & 14 \\
\hline HH - St. Georg & 656 & 403 & 105 & 128 & 20 & 49 & 11 \\
\hline HH - UKE & 689 & 128 & 214 & 286 & 61 & 46 & 23 \\
\hline Leipzig & 1192 & 418 & 109 & 607 & 58 & 28 & 22 \\
\hline Magdeburg & 487 & 223 & 87 & 42 & 135 & 13 & 8 \\
\hline Mannheim & 471 & 313 & 91 & 48 & 19 & 19 & 10 \\
\hline München & 788 & 197 & 348 & 130 & 113 & 40 & 15 \\
\hline Münster & 1700 & 428 & 845 & 341 & 86 & 41 & 26 \\
\hline Stuttgart & 789 & 457 & 29 & 192 & 111 & 34 & 18 \\
\hline & 9583 & 3785 & 2348 & 2639 & 811 & 380 & 194 \\
\hline
\end{tabular}

Tab. 2 AFNET/EHRA Konsensuskonferenzen

\begin{tabular}{llll}
\hline Konferenz & Thema & Datum & $\begin{array}{l}\text { Anzahl } \\
\text { Teilnehmer }\end{array}$ \\
\hline $\begin{array}{l}\text { 1. AFNET/ } \\
\text { EHRA-Konsensus- } \\
\text { konferenz }\end{array}$ & $\begin{array}{l}\text { Outcome parameters for atrial fibrillation } \\
\text { trials [43, 44] }\end{array}$ & 22.-23.01.2007 & 56 \\
\hline $\begin{array}{l}\text { 2. AFNET/ } \\
\text { EHRA-Konsensus- } \\
\text { konferenz }\end{array}$ & $\begin{array}{l}\text { Research perspectives for diagnosis and } \\
\text { treatment of atrial fibrillation [45, 46] }\end{array}$ & 27.-28.10.2008 & 68 \\
\hline $\begin{array}{l}\text { 3. AFNET/ } \\
\begin{array}{l}\text { EHRA-Konsensus- } \\
\text { konferenz }\end{array}\end{array}$ & $\begin{array}{l}\text { Stroke prevention, thromboembolic risk as- } \\
\text { sessment, modification of risk factors, and } \\
\text { rhyth control to reduce cardiovascular }\end{array}$ & & \\
\hline $\begin{array}{l}\text { 4. AFNET/ } \\
\text { EHRA-Konsensus- } \\
\text { konferenz }\end{array}$ & $\begin{array}{l}\text { Connecting pathophysiological and clinical } \\
\text { data for personalized AF management [48] }\end{array}$ & 24.-25.01.2013 & 71 \\
\hline $\begin{array}{l}\text { 5. AFNET/ } \\
\text { EHRA-Konsensus- } \\
\text { konferenz }\end{array}$ & $\begin{array}{l}\text { Understanding and eliminating inequalities } \\
\text { and barriers that prevent optimal treatment } \\
\text { of atrial fibrillation [49] }\end{array}$ & $22 .-23.01 .2015$ & 73 \\
\hline
\end{tabular}

Sartanen das Auftreten von Vorhofflimmern bei Patienten mit Bluthochdruck oder Herzinsuffizienz verhindern kann. $\mathrm{Ob}$ dies eine Behandlung mit solchen Substanzen für andere Patienten mit Vorhofflimmern rechtfertigt, war vor 10 Jahren völlig unklar. Die ANTIPAFStudie untersucht die Wirksamkeit von Olmesartan (Angiotensin-II-Rezeptorblocker) im Vergleich zu Placebo zur Verringerung von paroxysmalem Vorhofflimmern, innerhalb einer multizentrischen, randomisierten, placebokontrollierten, doppelblinden Studie. Die zentrale Studienhypothese ist, dass die

\section{Flec SL - AFNET 3}

Die Behandlung mit Antiarrhythmika ist weiterhin die häufigste Form der rhythmuserhaltenden Therapie von Vorhofflimmern. Während die individuelle Effektivität von Antiarrhythmika schwer vorhersagbar ist und u. a. im CATCHME-Konsortium die Auswahl von geeigneten Patienten verbessert werden soll, ist es bekannt, dass Rezidive von Vorhofflimmern gehäuft in den ersten Wochen nach einer Kardioversion auftreten [30]. Diese klinische Beobachtung lässt sich, zumindest teilweise, dadurch erklären, dass sich elektrische Veränderungen im Vorhof („electrical remodelling“) in den ersten Wochen nach Wiederherstellung des normalen Sinusrhythmus zurückbilden [31, 32]. Die Flec SL - AFNET 3 Studie hat deswegen geprüft, ob eine Kurzzeitbehandlung mit dem Antiarrhythmikum Flecainid für wenige Wochen nach Kardioversion ähnlich effektiv ist wie eine Langzeitbehandlung. Die Studie wurde in 44 AFNET-Zentren in Deutschland durchgeführt. Die Ergebnisse belegen einerseits die relative Effektivität der Kurzzeitbehandlung, die ca. $80 \%$ der Vorhofflimmerrezidive im Vergleich zu einer Langzeitbehandlung verhindert, weisen aber auch darauf hin, dass noch weitere Faktoren zu Vorhofflimmerrezidiven nach Kardioversion beitragen, die ebenfalls mittels Antiarrhythmika verhindert werden können [33]. Eine erste Subanalyse der Studiendaten bestätigte die Sicherheit der elektrischen Kardioversion [34]. Weitere Analysen der Studiendaten, u. a. durch EUTRAF- und CATCH-MEPartner, sind in Arbeit.

Neben diesen großen klinischen Projekten sind im AFNET weitere Projekte erfolgreich abgeschlossen worden, z. B. zur Detektion von kleinen potenziell ischämischen Läsionen in „asymptomatischen“ Patienten mit Vorhofflimmern [35], umfassende Analysen zur Pathophysiologie von Vorhofflimmern [2-4, $6,13,36,37]$ oder eine Patientenbroschüre, die inzwischen in dritter Auflage auf Papier und als PDF online vieltausendfach verwendet wurde (www. kompetenznetz-vorhofflimmern.de/de/ neue-patientenbroschuere-informiertueber-vorhofflimmern). 
Tab. 3 Aktuelle klinische Studien und Projekte im AFNET e.V.

\begin{tabular}{|c|c|c|c|c|c|c|}
\hline \multirow[b]{2}{*}{ Akronym } & \multirow[b]{2}{*}{ Ziel } & \multirow[b]{2}{*}{ Finanzierung } & \multicolumn{4}{|c|}{ Patientenzahlen } \\
\hline & & & Sponsor & Start & Ziel & $\begin{array}{l}\text { Stand } \\
10 / 2015\end{array}$ \\
\hline $\begin{array}{l}\text { EORP AF } \\
\text { (deut- } \\
\text { scher } \\
\text { Teil) }\end{array}$ & $\begin{array}{l}\text { Erfassung der Versor- } \\
\text { gung von Patienten } \\
\text { mit Vorhofflimmern } \\
\text { (inkl. Patienten, die } \\
\text { nicht von Kardiologen } \\
\text { gesehen werden) }\end{array}$ & $\begin{array}{l}\text { ESC, DZHK, } \\
\text { Industriepart- } \\
\text { ner }\end{array}$ & AFNET & Q4 2014 & 3500 & 1600 \\
\hline $\begin{array}{l}\text { EAST - } \\
\text { AFNET } 4\end{array}$ & $\begin{array}{l}\text { Prüfung des prognos- } \\
\text { tischen Werts einer } \\
\text { frühen rhythmuserhal- } \\
\text { tenden Behandlung }\end{array}$ & $\begin{array}{l}\text { EHRA, DZHK, } \\
\text { Herzstiftung, } \\
\text { Industriepart- } \\
\text { ner }\end{array}$ & AFNET & Q3 2011 & 2745 & 2100 \\
\hline $\begin{array}{l}\text { AXAFA - } \\
\text { AFNET } 5\end{array}$ & $\begin{array}{l}\text { Prüfung der Sicherheit } \\
\text { einer kontinuierlichen } \\
\text { Antikoagulation mit } \\
\text { NOACs während Ka- } \\
\text { theterablation von } \\
\text { Vorhofflimmern }\end{array}$ & $\begin{array}{l}\text { DZHK, Indu- } \\
\text { striepartner }\end{array}$ & AFNET & Q1 2015 & 650 & 90 \\
\hline $\begin{array}{l}\text { NOAH - } \\
\text { AFNET } 6\end{array}$ & $\begin{array}{l}\text { Prüfung des Nutzens } \\
\text { einer oralen Antikoa- } \\
\text { gulation bei Patienten } \\
\text { mit "atrial high rate } \\
\text { episodes" }\end{array}$ & $\begin{array}{l}\text { DZHK, Indu- } \\
\text { striepartner }\end{array}$ & AFNET & $\begin{array}{l}\text { Q1 } 2016 \\
\text { (ge- } \\
\text { plant) }\end{array}$ & 3400 & - \\
\hline $\begin{array}{l}\text { RE-LATED } \\
\text { - AFNET } 7\end{array}$ & $\begin{array}{l}\text { Prüfung der Wirksam- } \\
\text { keit eines NOAC für die } \\
\text { Auflösung von atrialen } \\
\text { Thromben }\end{array}$ & $\begin{array}{l}\text { Industriepart- } \\
\text { ner }\end{array}$ & $\begin{array}{l}\text { Universität } \\
\text { Mainz }\end{array}$ & Q3 2013 & 110 & 32 \\
\hline $\begin{array}{l}\text { MonDAFIS } \\
\text { (AFNET } \\
\text { associa- } \\
\text { ted) }\end{array}$ & $\begin{array}{l}\text { Prüfung der Relevanz } \\
\text { eines verlängerten } \\
\text { EKG-Monitorings für } \\
\text { die Detektion eines bis } \\
\text { dato nicht bekannten } \\
\text { paroxysmalen Vor- } \\
\text { hofflimmerns und die } \\
\text { konsekutive medika- } \\
\text { mentöse } \\
\text { Sekundärprävention } \\
\text { bei Patienten mit ak- } \\
\text { utem ischämischem } \\
\text { Schlaganfall }\end{array}$ & $\begin{array}{l}\text { Industriepart- } \\
\text { ner }\end{array}$ & $\begin{array}{l}\text { Charité - } \\
\text { Univer- } \\
\text { sitätsme- } \\
\text { dizin } \\
\text { Berlin }\end{array}$ & Q2 2014 & 3470 & 600 \\
\hline $\begin{array}{l}\text { DZHK Deut } \\
\text { ESC Europäi }\end{array}$ & ches Zentrum für Herz- & Kreislaufforsc & 9, AFINEI & trial Fibril & $n 1$ & $\begin{array}{l}\text { Jork, } \\
\text { Quartal }\end{array}$ \\
\hline
\end{tabular}

\section{AFNET-Partner in Deutschland und Europa}

Aufgrund der fachlichen Nähe pflegte das Kompetenznetz Vorhofflimmern einen sehr engen Informationsaustausch mit den beiden weiteren kardiologischen Kompetenznetzen „Angeborene Herzfehler" und "Herzinsuffizienz". Um die Sichtbarkeit der kardiologischen Netzwerke zu stärken, waren die kardiologischen Kompetenznetze jeweils mit einem Gemeinschaftsstand bei den Jahrestagungen der Deutschen Gesellschaft für Kardiologie (DGK) vertreten. Die kardiologischen Kompetenznetze waren zudem gemeinsam erfolgreich bei der Beantragung einer netzwerkspezifischen Förderung durch das DZHK. Um die Vernetzung auch fachübergreifend $\mathrm{zu}$ fördern und den Erfahrungsaustausch mit den anderen Kompetenznetzen in der Medizin zu intensivieren, haben sich Vertreter des Kompetenznetzes Vorhofflimmern an verschiedenen Arbeitsgruppen der "Technologie- und Methodenplattform für die vernetzte medizinische Forschung e. V." (TMF) beteiligt.

Im Rahmen der jährlichen Frühjahrstagung der Deutschen Gesellschaft für Kardiologie (DGK) hatte das AFNET bis 2014 jeweils eine eigene wissenschaftliche Sitzung, bei welcher die aktuellen Forschungsergebnisse intensiv diskutiert wurden. Durch die hohe Besucherzahl der Jahrestagungen konnte das AFNET im Rahmen seiner Sitzungen eine sehr gute Sichtbarkeit des Forschungsnetzes und seiner Ergebnisse gewährleisten. Zusammen mit den anderen kardiologischen Kompetenznetzen war das AFNET zudem mit einem Gemeinschaftsstand bei der Frühjahrstagung der DGK vertreten. Die besondere Anbindung an die Fachgesellschaft ergibt sich auch daraus, dass drei der bisherigen Mitglieder des Vorstandes Präsidenten der DGK waren; zudem war ein Mitglied Präsident der Europäischen Gesellschaft für Kardiologie. Während des gesamten Förderzeitraums war die Deutsche Herzstiftung als unabhängige, die Patienteninteressen vertretende Institution ein enger Kooperationspartner. Das AFNET beteiligte sich an verschiedenen Veranstaltungen der Deutschen Herzstiftung, zudem unterstützte die Deutsche Herzstiftung einzelne Aktivitäten des AFNET. Bei der Bereitstellung von Informationsmaterialien, z. B. der Patientenbroschüre „Herzrhythmusstörungen“ der Herzstiftung, arbeiteten AFNET und Deutsche Herzstiftung eng zusammen, um Patienten umfassend und unabhängig über das Krankheitsbild Vorhofflimmern zu informieren.

Das AFNET hat schon früh internationale Partnerschaften gesucht. Seit 2007 hat das AFNET gemeinsam mit der European Heart Rhythm Association (EHRA) 5 internationale AFNET/EHRA-Konsensuskonferenzen organisiert (siehe - Tab. 2). Diese Konsensuskonferenzen und die publizierten Ergebnisse sind vielbeachtet und haben Entwicklungen von „outcome parameters in clinical AF trials" bis hin zu Parametern zur Qualitätsmessung bei Vorhofflimmerbehandlungen definiert (siehe 0 Tab. 1). Eine 6. Konferenz ist geplant. Die Ergebnisse der Konsensuskonferenzen wurden als Empfehlungen zur Forschung, Diagnose und Therapie von Vorhofflimmern publiziert. Die Konferenzen dienten von Anfang an dazu, Fragen, die durch die Arbeit des Netzes entstanden waren, öf- 
Tab. 4 Lenkungsausschuss des Kompetenznetzes Vorhofflimmern e. V. Zusätzlich ist die Zentrale des AFNET e. V. im Lenkungsausschuss durch die Geschäftsführung vertreten

\begin{tabular}{|ll}
\hline Prof. Dr. med. Paulus Kirchhof (Vorstandsvorsitzender) & Birmingham, UK/Münster \\
\hline Prof. Dr. med. Andreas Goette (Vorstandsmitglied) & Paderborn \\
\hline $\begin{array}{l}\text { Prof. Dr. med. Michael Näbauer (Vorstandsmitglied, Schatzmeis- } \\
\text { ter) }\end{array}$ & München \\
\hline Prof. Dr. med. Dr. rer. nat. Ulrich Schotten (Vorstandsmitglied) & Maastricht, NL/Aachen \\
\hline Univ.- Prof. em. Dr. Dr. h.c. Günter Breithardt & Münster \\
\hline Prof. Dr. med. Lars Eckardt & Münster \\
\hline PD Dr. med. Karl Georg Häusler & Berlin \\
\hline Prof. Dr. med. Thorsten Lewalter & München \\
\hline Prof. Dr. med. Thomas Meinertz & Hamburg \\
\hline Prof. Dr. med. Michael Oeff & Brandenburg \\
\hline Prof. Dr. med. Dr. h.c. Ursula Ravens & Dresden \\
\hline Prof. Dr. med. Thomas Rostock & Mainz \\
\hline Prof. em. Dr. med. Gerhard Steinbeck & München \\
\hline Prof. Dr. rer. pol. Karl Wegscheider & Hamburg \\
\hline Ausgeschiedene Mitglieder & \\
\hline Prof. Dr. med. Peter Hanrath (ehemaliges Vorstandsmitglied) & Aachen \\
\hline Prof. Dr. Walter Lehmacher & Köln \\
\hline
\end{tabular}

fentlich und zielorientiert zu diskutieren und nach bestem Stand des Wissens $\mathrm{zu}$ klären. EHRA ist zudem wichtiger wissenschaftlicher Partner bei der Durchführung kontrollierter internationaler klinischer Studien, etwa als Partner des AFNET e. V. bei der Durchführung von EAST - AFNET 4 oder bei NOAH - AFNET 6.

Die Jahrestagungen der EHRA und der europäischen Gesellschaft für Kardiologie (ESC, www.escardio.org) sind wichtige Kommunikationsplattformen für das AFNET auf europäischer Ebene. Mitglieder des AFNET waren wesentlich an der Erstellung der Leitlinien zum Thema Vorhofflimmern der ESC beteiligt [38-41]. Die Ergebnisse von ANTIPAF - AFNET 2 [29] und Flec SL - AFNET 3 [33] sind direkt in diese Leitlinien eingeflossen, die die Behandlung von Vorhofflimmern in über 50 Ländern Europas regeln und die weltweit Beachtung finden. In Deutschland, wie in vielen anderen Ländern, werden die ESC-Leitlinien von den nationalen kardiologischen Gesellschaften adoptiert und kommentiert.

Seit 2015 ist das DZHK ein wesentlicher Partner des AFNET. Das DZHK unterstützt das AFNET bei der Durchführung mehrerer laufender Studien (siehe Tab. 3).

\section{Verstetigung des AFNET: eingetragener Verein, Entwicklung zur Academic Research Organisation}

Zur Sicherung dieser Erfolge hat das Kompetenznetz Vorhofflimmern bereits im Jahr 2010 den Verein Kompetenznetz Vorhofflimmern e. V. gegründet, um die Arbeit außerhalb Deutschlands besser zu unterstützen und um eine Fortsetzung der Arbeit nach Ende der Förderung durch das BMBF zu ermöglichen. Der Verein hat seinen Sitz in Münster, wo auch die Zentrale des AFNET mit 6 Vollzeitmitarbeitern angesiedelt ist. Der Verein wird, wie die BMBF-geförderte Struktur, durch einen Vorstand und Lenkungsausschuss gesteuert. Zudem wird die Arbeit des AFNET e. V. durch einen Beirat begleitet und überwacht. Die Gründung des Vereins ermöglichte die Initiierung von internationalen klinischen Studien, deren Sponsor der Verein ist. Die EAST - AFNET 4 Studie [42] war das erste dieser Projekte (siehe - Tab. 3). Die Wahrnehmung dieser Funktion, die essenziell für die Durchführung von unabhängigen klinischen Studien ist, ist an Anforderungen gebunden, die von einer unabhängigen Organisation leichter erfüllt werden können.

\section{Laufende Projekte}

Aktuell ist das AFNET an mehreren großen klinischen Projekten beteiligt. Hervorzuheben sind insbesondere die internationalen klinischen Studien EAST - AFNET 4, AXAFA - AFNET 5 und NOAH - AFNET 6, bei denen AFNET e.V. als Sponsor im gesetzlichen Sinne fungiert. AFNET e.V. ist der von der deutschen Gesellschaft für Kardiologie beauftragte Partner der ESC für die Durchführung des EORP Atrial Fibrillation Registers. Alle Studien sind investigatorinitiierte Projekte und werden in Mischfinanzierung aus öffentlicher Förderung und durch Industriepartner gefördert. AFNET ist Partner eines europäischen Konsortiums mit dem Ziel, klinische Marker für verschiedene Formen von Vorhofflimmern zu entwickeln (CATCH ME, s. Abschn. „Aufbau europäischer und internationaler Forschungskooperationen"). In diesen Studien werden systematisch Bioproben gesammelt, sodass die Datensätze für explorative Analysen nach Studienende zur Verfügung gestellt werden können. AFNET ist zudem an weiteren klinischen Studien, etwa MonDAFIS und RE-LATED - AFNET 7, beteiligt. AFNET e. V. hat sich zu einer Plattform entwickelt, die die Durchführung von investigatorinitiierten klinischen Studien im kardiovaskulären Bereich in Deutschland und in Europa ermöglicht. Wir hoffen, dass das AFNET so weiter zur Verbesserung der Forschungslandschaft in Deutschland und zu einer besseren Versorgung von Patienten mit Rhythmusstörungen und anderen kardiovaskulären Erkrankungen beitragen kann.

\section{Korrespondenzadresse}

Professor Dr. med. P. Kirchhof

Kompetenznetz Vorhofflimmern

Mendelstraße 11, 48149 Münster, Deutschland info@kompetenznetz-vorhofflimmern.de p.kirchhof@bham.ac.uk

Danksagung. Die Autoren, der aktuelle Vorstand des AFNET e. V., möchte allen ehemaligen und aktuellen Vorstandsmitgliedern und Lenkungsausschussmitgliedern für ihren Beitrag zum hier beschriebenen gemeinsamen Erfolg danken (siehe - Tab. 4). Stellvertretend für die vielen Mitarbeiterinnen und Mitarbeiter möchten wir besonders den 
beiden ehemaligen Geschäftsführerinnen und Geschäftsführern des AFNET, Frau Dr. Gerlinde Benninger und Herrn Dr. Thomas Weiss, für ihr Engagement danken. Vor allem aber gilt unser Dank den Mitgliedern im Verein, den Studienzentren und den vielen Personen, die sich an Untersuchungen des AFNET beteiligt haben und beteiligen.

\section{Einhaltung ethischer Richtlinien}

Interessenkonflikt. Der vorliegende Beitrag wurde von den Vorstandsmitgliedern des Kompetenznetzes Vorhofflimmern e. V. verfasst (AFNET e. V). P. Kirchhof, A. Goette, M. Näbauer und U. Schotten geben an, dass kein Interessenkonflikt besteht.

Dieser Beitrag beinhaltet keine Studien an Menschen oder Tieren.

Open Access. This article is distributed under the terms of the Creative Commons Attribution 4.0 International License (http://creativecommons.org/ licenses/by/4.0/), which permits unrestricted use, distribution, and reproduction in any medium, provided you give appropriate credit to the original author(s) and the source, provide a link to the Creative Commons license, and indicate if changes were made.

\section{Literatur}

1. Kirchhof P, Schotten U (2006) Hypertension begets hypertrophy begets atrial fibrillation? Insights from yet another sheep model. Eur Heart J 27(24):2919-2920

2. Schotten U, Dobrev $D$, Kirchhof $P$ et al (2006) Vorhofflimmern: Grundlagenforschung liefert neue Therapieansätze. Dtsch Arztebl 103(25):A1743-A1748

3. Kirchhof P, Kahr PC, Kaese $S$ et al (2011) PITX2c is expressed in the adult left atrium, and reducing Pitx2c expression promotes atrial fibrillation inducibility and complex changes in gene expression. Circ Cardiovasc Genet 4(2):123-133

4. Schotten U, Verheule S, Kirchhof P et al (2011) Pathophysiological mechanisms of atrial fibrillation: a translational appraisal. Physiol Rev 91(1):265-325

5. Wakili R, Yeh YH, Yan Qi X et al (2010) Multiple potentialmolecularcontributors toatrial hypocontractility caused by atrial tachycardia remodeling in dogs. Circ Arrhythm Electrophysiol 3(5):530-541

6. Kirchhof P, Marijon E, Fabritz L et al (2013) Overexpression of CAMP-response element modulator causes abnormal growth and development of the atrial myocardium resulting in a substrate for sustained atrial fibrillation in mice. Int J Cardiol 166(2):366-374

7. Christ T, Boknik P, Wohrl S et al (2004) L-type $\mathrm{Ca} 2+$ current downregulation in chronic human atrial fibrillation is associated with increased activity of protein phosphatases. Circulation 110(17):2651-2657

8. Gaborit N, Steenman M, Lamirault G et al (2005) Human atrial ion channel and transporter subunit gene-expression remodeling associated with valvular heart disease and atrial fibrillation. Circulation 112(4):471-481

9. Vest JA, Wehrens XH, Reiken SR et al (2005) Defective cardiac ryanodine receptor regulation during atrial fibrillation. Circulation 111(16):2025-2032
10. El-Armouche A, Boknik $P$, Eschenhagen $T$ et al (2006) Molecular determinants of altered Ca2+ handling in human chronic atrial fibrillation. Circulation 114(7):670-680

11. Voigt N, Maguy A, Yeh YHet al (2008) Changes in IK, ACh single-channel activity with atrial tachycardia remodelling in canine atrial cardiomyocytes. Cardiovasc Res 77(1):35-43

12. Chelu MG, Satyam S, Sood Setal (2009) Calmodulin kinase II-mediated sarcoplasmatic reticulum $\mathrm{Ca} 2+$ leak promotes atrial fibrillation in mice. J Clin Invest 119(7):1940-1951

13. Goette A, Bukowska A, Dobrev D et al (2009) Acute atrial tachyarrhythmia induces angiotensin Il type 1 receptor-mediated oxidative stress and microvascular flow abnormalities in the ventricles. Eur Heart J30(11):1411-1420

14. Dobrev D, Nattel S (2010) New antiarrhythmic drugs for treatment of atrial fibrillation. Lancet 375(9721):1212-1223

15. Pfeufer A, van Noord C, Marciante KD et al (2010) Genome-wide association study of PR interval. Nat Genet 42(2):153-159

16. den Hoed M, Eijgelsheim M, Esko T et al (2013) Identification of heart rate-associated loci and their effects on cardiac conduction and rhythm disorders. Nat Genet 45(6):621-631

17. Lin H, Sinner MF, Brody JA et al (2014) Targeted sequencing in candidate genes for atrial fibrillation: the Cohorts for Heart and Aging Research in Genomic Epidemiology (CHARGE) Targeted Sequencing Study. Heart Rhythm 11(3):452-457

18. Benjamin EJ, Rice KM, Arking DE et al (2009) Variants in ZFHX3 are associated with atrial fibrillation in individuals of European ancestry. Nat Genet 41(8):879-881

19. Kaab S, Darbar D, van Noord C et al (2009) Large scale replication and meta-analysis of variants on chromosome $4 \mathrm{q} 25$ associated with atrial fibrillation. Eur Heart J 30(7):813-819

20. Ellinor PT, Lunetta KL, Glazer NL et al (2010) Common variants in KCNN3 are associated with lone atrial fibrillation. Nat Genet 42(3):240-244

21. Lubitz SA, Sinner MF, Lunetta KL et al (2010) Independent susceptibility markers for atrial fibrillation on chromosome $4 q 25$. Circulation 122(10):976-984

22. Ellinor PT, Lunetta KL, Albert CM et al (2012) Metaanalysis identifies six new susceptibility loci for atrial fibrillation. Nat Genet 44(6):670-675

23. Breithardt G, Dobrev D, Doll N et al (2008) The German Competence Network on Atrial Fibrillation (AFNET). Herz 33(8):548-555

24. Nabauer M, Gerth A, Limbourg T et al (2009) The Registry of the German Competence NETwork on Atrial Fibrillation: Patient characteristics and initial management. Europace 11:423-434

25. Kirchhof $P$, Nabauer M, Gerth A et al (2011) Impact of the type of centre on management of AF patients: surprising evidence for differences in antithrombotic therapy decisions. Thromb Haemost 105(6):1010-1023

26. Reinecke H, Nabauer M, Gerth A et al (2015) Morbidity and treatment in patients with atrial fibrillation and chronic kidney disease. Kidney Int 87:200-209

27. Calkins H, Kuck KH, Cappato R et al (2012) HRS/ EHRA/ECAS Expert Consensus Statement on Catheter and Surgical Ablation of Atrial Fibrillation: recommendations for patient selection, procedural techniques, patient management and follow-up, definitions, endpoints, and research trial design. Europace 14(4):528-606
28. Goette A, Breithardt G, Fetsch T et al (2007) Angiotensin II antagonist in paroxysmal atrial fibrillation (ANTIPAF) trial: rationale and study design. Clin Drug Investig 27(10):697-705

29. Goette A, Schon N, Kirchhof P et al (2012) Angiotensin Il-antagonist in paroxysmal atrial fibrillation (ANTIPAF) trial. Circ Arrhythm Electrophysiol 5(1):43-51

30. KirchhofP, Fetsch T, Hanrath P et al (2005) Targeted pharmacological reversal of electrical remodeling after cardioversion - rationale and design of the Flecainide Short-Long (Flec-SL) trial. Am Heart J 150(5):899-e1-e6

31. Kirchhof $P$, Engelen M, Franz MR et al (2005) Electrophysiological effects of flecainide and sotalol in the human atrium during persistent atrial fibrillation. Basic Res Cardiol 100(2):112-121

32. Wijffels MC, Kirchhof CJ, Dorland R et al (1995) Atrial fibrillation begets atrial fibrillation. A study in awake chronically instrumented goats. Circulation 92(7):1954-1968

33. Kirchhof P, Andresen D, Bosch R et al (2012) Short-term versus long-term antiarrhythmic drug treatment after cardioversion of atrial fibrillation (Flec-SL): a prospective, randomised, openlabel, blinded endpoint assessment trial. Lancet 380(9838):238-246

34. Apostolakis S, Haeusler KG, Oeff M et al (2013) Low stroke risk after elective cardioversion of atrial fibrillation: an analysis of the Flec-SL trial. Int J Cardiol 168(4):3977-3981

35. Knecht S, Oelschlager C, Duning Tet al (2008) Atrial fibrillation in stroke-free patients is associated with memory impairment and hippocampal atrophy. Eur Heart J 29(17):2125-2132

36. Wakili R, Voigt N, Kaab S, Dobrev D, Nattel $S$ (2011) Recent advances in the molecular pathophysiology of atrial fibrillation. J Clin Invest 121(8):2955-2968

37. Makary S, Voigt N, Maguy A et al (2011) Differential protein kinase $\mathrm{C}$ isoform regulation and increased constitutive activity of acetylcholine-regulated potassium channels in atrial remodeling. Circ Res 109(9):1031-1043

38. Camm AJ, Lip GY, De Caterina R et al (2012) 2012 focused update of the ESC Guidelines for the management of atrial fibrillation: an update of the 2010 ESC Guidelines for the management of atrial fibrillation-developed with the special contribution of the European Heart Rhythm Association. Europace 14(10):1385-1413

39. Camm AJ, Lip GY, De Caterina R et al (2012) 2012 focused update of the ESC Guidelines for the management of atrial fibrillation: An update of the 2010 ESC Guidelines for the management of atrial fibrillation * Developed with the specia contribution of the European Heart Rhythm Association. Eur Heart J 33(21):2719-2747

40. Kirchhof $P$, Curtis AB, Skanes AC et al (2013) Atrial fibrillation guidelines across the Atlantic: a comparison of the current recommendations of the European Society of Cardiology/European Heart Rhythm Association/European Association of Cardiothoracic Surgeons, the American College of Cardiology Foundation/American Heart Association/Heart Rhythm Society, and the Canadian Cardiovascular Society. Eur Heart J 234(20):1471-1474

41. Camm AJ, KirchhofP, Lip GY et al (2010) Guidelines for the management of atrial fibrillation: the Task Force for the Management of Atrial Fibrillation of the European Society of Cardiology (ESC). Europace 12(10):1360-1420 


\section{Leitthema}

42. Kirchhof P, Breithardt G, Camm AJ et al (2013) Improving outcomes in patients with atrial fibrillation: rationale and design of the Early treatment of Atrial fibrillation for Stroke prevention Trial. Am Heart J 166(3):442-448

43. Kirchhof P, Auricchio A, Bax J et al (2007) Outcome parameters for trials in atrial fibrillation: Recommendations from a consensus conference organized by the German Atrial Fibrillation Competence NETwork and the European Heart Rhythm Association. Europace 9:1006-1023

44. Kirchhof $P$, Auricchio A, Bax J et al (2007) Outcome parameters for trials in atrial fibrillation: executive summary: Recommendations from a consensus conference organized by the German Atrial Fibrillation Competence NETwork (AFNET) and the European Heart Rhythm Association (EHRA. Eur Heart J 28(22):2803-2817

45. Kirchhof $P$, Bax J, Blomstrom-Lundquist $C$ et al (2009) Early and comprehensive management of atrial fibrillation: proceedings from the 2 nd AFNET/ EHRA consensus conference on atrial fibrillation entitled 'research perspectives in atrial fibrillation'. Europace 11(7):860-885

46. Kirchhof $\mathrm{P}, \mathrm{Bax} \mathrm{J}$, Blomstrom-Lundquist $\mathrm{C}$ et al (2009) Early and comprehensive management of atrial fibrillation: executive summary of the proceedings from the 2 nd AFNET-EHRA consensus conference 'research perspectives in AF'. Eur Heart J30(24):2969-2977c

47. Kirchhof P, Lip GY, Van Gelder IC et al (2011) Comprehensive risk reduction in patients with atrial fibrillation: Emerging diagnostic and therapeutic options. Executive summary of the report from the 3rd AFNET/EHRA consensus conference. Thromb Haemost 106(6):1012-1019

48. Kirchhof P, Breithardt G, Aliot E et al (2013) Personalized management of atrial fibrillation: Proceedings from the fourth Atrial Fibrillation competence NETwork/European Heart Rhythm Association consensus conference. Europace 15(11):1540-1556

49. Kirchhof $P$, Breithardt $G$, Bax J, Benninger $G$, Blomstrom-Lundqvist $C$, Boriani $G$, Brandes $A$, Brown $\mathrm{H}$, Brueckmann $M$, Calkins $\mathrm{H}$, Calvert $\mathrm{M}$, Christoffels V, Crijns H, Dobrev D, Ellinor P, Fabritz L, Fetsch T, Freedman SB, Gerth A, Goette A, Guasch E, Hack G, Haegeli L, Hatem S, Haeusler KG, Heidbüchel H, Heinrich-Nols J, Hidden-Lucet $F$, Hindricks G, Juul-Möller S, Kääb S, Kappenberger L, KespohI S, Kotecha D, Lane DA, Leute A, Lewalter T, Meyer R, Mont L, Münzel F, Nabauer M, Nielsen JC, Oeff M, Oldgren J, Oto A, Piccini JP, Pilmeyer A, Potpara T, Ravens U, Reinecke $\mathrm{H}$, Rostock $\mathrm{T}$, Rustige J, Savelieva I, Schnabel R, Schotten U, Schwichtenberg L, Sinner MF, Steinbeck G, Stol M, Tavazzi L, Themistoclakis S, Tse HF, Van Gelder IC, Vardas PE, Varpula T, Vincent A, Werring D, Willems S, Ziegler A, Lip GY, Camm AJ (2015) A roadmap to improve the quality of atrial fibrillation management: Proceedings from the 5th AFNET/ EHRA consensus conference. Europace 2015. doi: 10.1093/europace/euv304 